\title{
Moving Mountains: Reshaping the Activity Volcano of Electrocatalysis with Fluxional Subnano Clusters
}

Zisheng Zhang 1 , Borna Zandkarimi ${ }^{1}$, Julen Munarriz ${ }^{1, \dagger}$, Claire Dickerson ${ }^{1}$, Anastassia N. Alexandova ${ }^{1,2, *}$

${ }^{1}$ Department of Chemistry and Biochemistry, University of California, Los Angeles, 607 Charles E. Young Drive, Los Angeles, California 90095-1569, United States

2 California NanoSystems Institute, Los Angeles, California 90095-1569, United States 
ABSTRACT. The activity volcano derived from Sabatier analysis provides intuitive guide for catalyst design, but it also imposes fundamental limitations on the maximal activity and the pool of high-performance elements. Here we show that the activity volcano for oxygen reduction reaction (ORR) can be shifted and reshaped in the subnano regime. The fluxional behavior of subnano clusters, in both isolated and graphite-supported forms, not only breaks the linear scaling relationships but also causes an overall strengthening in adsorbate binding. The metals with optimal adsorbate binding in the bulk form (Pt/Pd) thus suffer over-binding issues, while the metals that under-bind in the bulk form $(\mathrm{Ag} / \mathrm{Au})$ gain optimal reaction energetics. In addition, the potential-dependence of isomer energies differ, causing non-linear reaction free energy-potential relations and enabling populationtuning of specific isomers, thereby surpassing the apex of the activity volcano. The shift of the volcano that puts under-binding elements closer to the top is likely general in fluxional cluster catalysis, and can be used for cluster catalyst design. 
TOC GRAPHICS

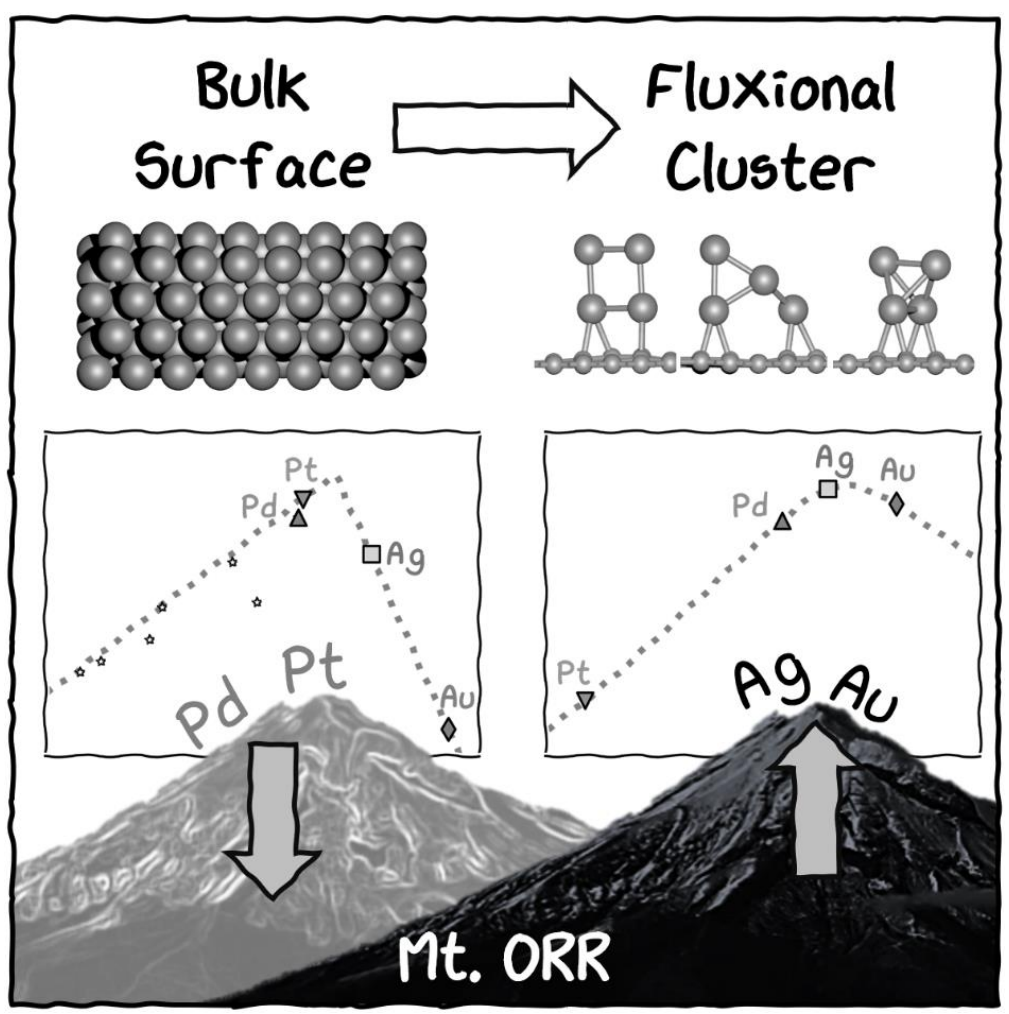

KEYWORDS Cluster catalysis, Fluxionality, Electrochemistry, Sabatier Principle, Oxygen reduction reaction 


\section{INTRODUCTION}

Activity volcano, based on the Sabatier principle and the scaling relationships of adsorption energetics, has been a time-tested model for catalyst design in the field of heterogeneous thermocatalysis and electrocatalysis. ${ }^{1,2}$ It states that the catalyst with maximal activity should have an intermediate and optimal binding strength with reactants, or else the activation or product desorption step will become rate-limiting due to under- or over-binding. Although the activity volcano model provides a simple and intuitive picture for optimizing catalysts, it poses an intrinsic limitation on the maximal theoretical activity for a given reaction. ${ }^{3}$

Subnano clusters have been demonstrated to have not only higher atomic efficiency but also fluxionality that set them apart from their bulk counterparts. ${ }^{4}$ Their relatively flat potential energy surface (PES) make them exceptionally dynamic. At finite temperature, subnano clusters can visit the low-energy local minima on the PES, populating a pool of coexisting isomers with distinct structures and reactivities, and that calls for a statistical ensemble representation. ${ }^{5}$ In addition, subnano clusters can adapt their core shapes to bind different adsorbates in different binding modes, and hence with different strengths. This leads to the breakdown of the scaling relationship (which normally assumes consistent binding mode among chemically similar adsorbates), and even potential nonArrhenius behavior of reaction kinetics. ${ }^{6,7}$ 
Oxygen reduction reaction $(\mathrm{ORR})$ is a well-studied electrocatalytic reaction where a robust scaling relationship and an activity volcano are observed for bulk metal surfaces. ${ }^{8}$ Since the scaling relationships underly the regular shape of the ORR activity volcano, we would expect the fluxionality and dynamics of subnano clusters to undermine the volcano. In this study, we explore how the ORR activity volcano looks like in the subnano regime. We show that the fluxionality and undercoordination of atoms in subnano cluster causes strengthening of adsorption, to a varying degree for different metals. This leads to the metals that under-bind in the bulk to acquire near-optimal adsorption energetics. As a result, the apex of the ORR activity volcano shifts from $\mathrm{Pt}$ and $\mathrm{Pd}$ to $\mathrm{Au}$ and Ag. The phenomenon is expected to be generalizable to other dynamic or restructuring systems, and that opens a new degree of freedom in catalyst design and make possible utilization of the elements that are usually regarded inert.

\section{RESULTS AND DISCUSSION}

For ORR, we consider the 4-electron dissociative and associative pathways which involve ${ }^{*} \mathrm{O},{ }^{*} \mathrm{OH}$, and ${ }^{*} \mathrm{OOH}$ intermediates $(*$ represents the catalyst's active site). The calculated binding energies $\Delta E_{\mathrm{O}}, \Delta E_{\mathrm{OH}}$, and $\Delta E_{\mathrm{OOH}}$ are referenced against the energies of $\mathrm{H}_{2} \mathrm{O}$ and $\mathrm{H}_{2}$ by corresponding stoichiometries. For the (111) surfaces of bulk metals, the binding energy of the three adsorbates correlate, and $E_{0}$ is often used to estimate the binding energies of other adsorbates and activation barriers. ${ }^{8}$ 
In this work we focus on $\mathrm{Pd}, \mathrm{Pt}, \mathrm{Ag}$, and $\mathrm{Au}$, a set that includes the two most active elements on both arms of the ORR activity volcano for bulk (111) surfaces. ${ }^{8}$ First, we study the subnano clusters $\mathrm{M}_{\mathrm{n}}(\mathrm{M}=\mathrm{Pd}, \mathrm{Pt}, \mathrm{Ag}, \mathrm{Au} ; \mathrm{n}=1-6)$ in the isolated form. A statistical ensemble of isomers is constructed for each bare and adsorbate-bound cluster via global optimization with bond length distribution algorithm (BLDA). ${ }^{9}$ The adsorbate binding energies are calculated using ensemble-average energies by Boltzmann populations at $300 \mathrm{~K}$ corresponding to the room temperature fuel cells. ${ }^{10}$ 

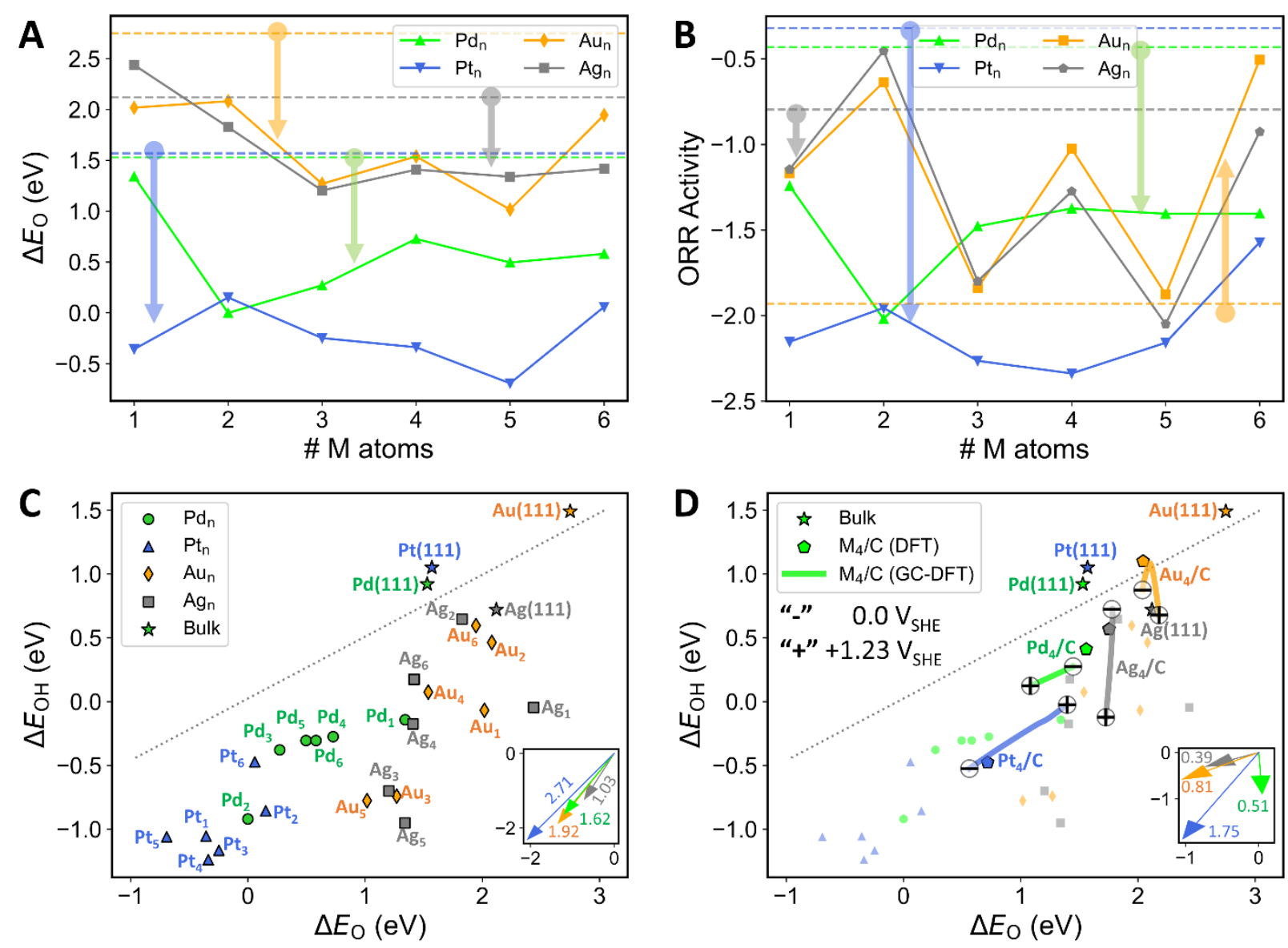

Figure 1. ORR energetics of isolated and graphite-supported clusters. (A) Binding energy for ${ }^{*} \mathrm{O}$ and (B) ORR activity of gas phase $\mathrm{Pd}_{\mathrm{n}}, \mathrm{Pt}_{\mathrm{n}}, \mathrm{Au}_{\mathrm{n}}, \mathrm{Ag}_{\mathrm{n}}$ clusters with $\mathrm{n}=1-6$. Values of bulk (111) surfaces are shown in dashed lines. The distribution of ${ }^{*} \mathrm{OH}$ and ${ }^{*} \mathrm{O}$ binding energies of (C) isolated and (D) graphite-supported clusters. The scaling relationship for bulk (111) is shown as dotted lines. "+" and "“" label the $0 \mathrm{~V}_{\mathrm{SHE}}$ and $1.23 \mathrm{~V}_{\mathrm{SHE}}$ values in the GCDFT curve. Insets show the shift from bulk (111) values to $\mathrm{M}_{1-6}$ values (averaged for each element, in $\mathrm{c}$ ) and $\mathrm{M}_{4} / \mathrm{C}$ values (in $\mathrm{d}$ ). 
In Figure 1a, we can see that the isolated clusters have an overall stronger binding of $\mathrm{O}$, by $0.5-2.0 \mathrm{eV}$ compared to the bulk surfaces (marked by dashed lines of corresponding colors). The binding strengthening originates in the undercoordination of atoms in subnano clusters, as well as fluxionality enabling them to rearrange in response of adsorbates thus maximizing the binding. As a result, $\Delta E_{\mathrm{O}}$ of $A u_{\mathrm{n}}$ and $\mathrm{Ag}_{\mathrm{n}}$ clusters strengthens to around $1.5 \mathrm{eV}$, which is close to the $\Delta E_{\mathrm{O}}$ of bulk $\mathrm{Pt}(111)$ and $\mathrm{Pd}(111) . \mathrm{Pt}_{\mathrm{n}}$ and $\mathrm{Pd}_{\mathrm{n}}$, however, shift to the over-binding regime.

The shifting in $\Delta E_{O}$ inspires us to investigate the ORR activity of those metals in the subnano cluster form. Figure $1 \mathrm{~b}$ shows the ORR activity of $\mathrm{M}_{n}$ calculated using an adapted microkinetic model from ref ${ }^{8}$ :

$$
A=k_{\mathrm{B}} T \min _{i}\left[\log \left(\frac{k_{i}}{k_{0}}\right)\right] ; \quad k_{i}=\nu_{i} \exp \left(-\frac{\Delta G_{i}}{k_{\mathrm{B}} T}\right)
$$

Where the pre-factors are taken from the original paper, but energies are replaced with computed ensemble-averaged values. Surprisingly, some of the $\mathrm{Au}_{\mathrm{n}}$ and $\mathrm{Ag} \mathrm{g}_{\mathrm{n}}$, such as $\mathrm{Ag}_{2}$ and $\mathrm{Au}_{6}$, gain an activity comparable to that of $\mathrm{Pd}(111)$, whereas the activity of $\operatorname{Pd}_{\mathrm{n}}$ and $\mathrm{Pt}_{\mathrm{n}}$ falls below the values of $\mathrm{Ag}(111)$ and $\mathrm{Au}(111)$, respectively.

Notably, there is no monotonic relationship between cluster size and binding energies (or activity) for subnano clusters, nor is the trend consistent among different metals. For nanoparticles, one could resort to the surface-to-volume ratio for a simple and intuitive 
explanation of size-dependence. In contrast, in the subnano regime, "each atom counts" in a non-trivial way. ${ }^{11,12}$

Figure 1c shows the $\Delta E_{\mathrm{OH}}-\Delta E_{\mathrm{O}}$ relation of $\mathrm{Pd}, \mathrm{Pt}, \mathrm{Ag}$, and $\mathrm{Au}$, in their bulk(111) and isolated cluster forms. Notably, selected $\mathrm{Ag}$ and Au clusters fall close to bulk Pt(111) and $\operatorname{Pd}(111)$, whereas Pt and Pd cluster clearly over-bind both adsorbates. The linear scaling relationship between ${ }^{*} \mathrm{OH}$ and ${ }^{*} \mathrm{O}$ adsorption energies break down for subnano clusters with a poor $R^{2}=0.60$ (versus $R^{2}=0.91$ for bulk surfaces) due to adsorbate-specific isomerization of the core structure as is discussed also in ref ${ }^{6}$. Compared to their bulk(111) values, the datapoints of isolated clusters overall shifts toward the lower left region due to strengthening of $\mathrm{O}$ and $\mathrm{OH}$ binding. The shift is larger for $\Delta E_{\mathrm{OH}}$ than for $\Delta E_{\mathrm{O}}$, likely due to more significant change in adsorbate configuration: $\mathrm{O}$ adsorbs majorly at bridge site both on bulk (111) and subnano clusters, whereas $\mathrm{OH}$ majorly adsorbs at atop site on bulk (111) but at bridge site on subnano clusters. This exploration qualitatively informs about the direction of the volcano reshaping for clusters. However, it can be expected that the drastic deviations seen for clusters would be tempered by deposition onto the cathode in a practical device, explored next.

We study the $\mathrm{M}_{4}$ supported on carbon substrate (denoted as $\mathrm{M}_{4} / \mathrm{C}$ ). The size of 4 atoms is chosen for its significant fluxionality, relatively small and therefore computationally manageable configurational space, and well-defined isomer shapes. The carbon substrate is modeled by a graphite slab (see section Methods), which could represent the surface of 
glassy carbon electrode or any graphitic carbon substrate. ${ }^{13}$ The ensemble-averaged cluster-support interaction energies in the absence of the applied potential are calculated to be $-1.92 \mathrm{eV},-1.30 \mathrm{eV},-1.03 \mathrm{eV}$, and $-0.64 \mathrm{eV}$ for $\mathrm{Pd}, \mathrm{Pt}, \mathrm{Ag}$, and $\mathrm{Au}$, respectively. The strongest cluster-support binding for $\mathrm{Pt}_{4} / \mathrm{C}$ flips its isomer distribution at $300 \mathrm{~K}$ : from the butterfly structure being prevalent over the tetrahedral in the gas phase, to the tetrahedron being prevalent over the butterfly on the surface. For the other three metals, the distribution is almost unchanged between isolated and supported forms (Figure S1). $\mathrm{M}_{4} / \mathrm{C}$ systems feature stronger binding of the adsorbates compared to their bulk (111), but the shifts are, expectedly, smaller compared to those of isolated subnano clusters (Figure 1d). The shift directions diverge for different $\mathrm{M}_{4} / \mathrm{C}$ systems (Figure $1 \mathrm{~d}$ inset), likely attributable to the competition between intra-cluster, cluster-support, and clusteradsorbate interactions. For example, for Pt, the change of the global minimum (GM) is due to the cluster-support interaction outcompeting the intra-cluster metal-metal interactions. The competition among different interactions can also be observed in the pair-correlation heatmap in Figure S2 - intuitively placing properties of clusters between those of the bulk and isolated atoms. For example, $\Delta E_{\mathrm{O}}$ of bulk (111) correlates with the Brinell hardness, which is an empirical measure of the bulk property. However, $\Delta E_{0}$ of clusters correlates better with atomic-related properties such as bond dissociation energy of the M-O dimer, cohesive energy of the bulk, and ionization energy. 
At a realistic electrochemical interface, the binding energy of adsorbate is potentialdependent and configuration-specific. ${ }^{14,15}$ For subnano clusters, the isomers in the ensemble can differ significantly in their redox properties and polarizabilities, thus reshaping the PES as the electrode potential varies. While it has been shown that high temperatures can change the Boltzmann population of fluxional clusters, here the electrode potential can significantly affect the populations even at low temperatures.

Take the $\mathrm{Pt}_{4} / \mathrm{C}$ system as an example: Figure 2 shows the potential-dependent isomer distribution for each ORR intermediate (Boltzmann statistics, $300 \mathrm{~K}$ ), calculated from grand canonical DFT (GCDFT) electronic free energies. For the bare $\mathrm{Pt}_{4} / \mathrm{C}$ at low overpotential, the square configuration is favored, but as the potential sweeps across c.a. 1.0 $\mathrm{V}$ in the SHE scale (denoted as $\mathrm{V}_{\mathrm{SHE}}$ ), the tetrahedron configuration becomes dominant (Figure 2a). For the $* \mathrm{O}$ intermediate (Figure 2b), however, the square configuration is inaccessible, and instead the tetrahedron, butterfly, and triangle-stick configurations coexist at low overpotential. At higher overpotential, the triangle-stick configuration becomes dominant. For the ${ }^{*} \mathrm{OH}$ intermediate (Figure 2c), the butterfly configuration is inaccessible, and the low and high overpotential regions are dominated by tetrahedron and triangle-stick configurations, respectively. For the ${ }^{*} \mathrm{OOH}$ intermediate (Figure 2d), the potential-dependent ensemble gets more complicated, with the butterfly, tetrahedron, and square configurations dominating the low, medium, and high overpotentials, respectively. The analyses for $\mathrm{Pd}, \mathrm{Au}$, and $\mathrm{Ag}$ are given in Figures S4-S6. 

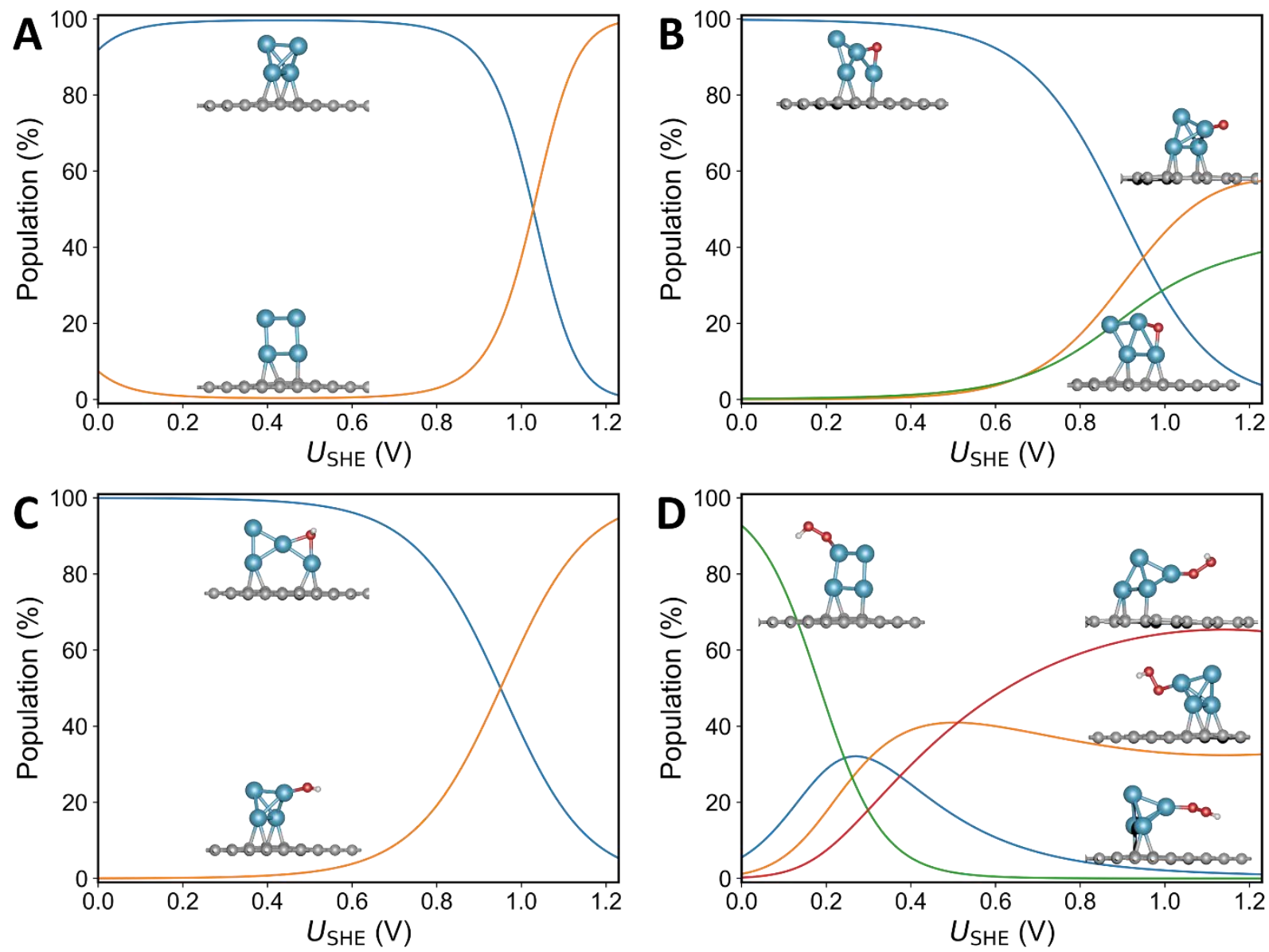

Figure 2. Potential-dependent isomer distributions of (a) *, (b) * $\mathrm{O},(\mathrm{c}){ }^{*} \mathrm{OH}$, and (d) *OOH intermediates on $\mathrm{Pt}_{4} / \mathrm{C}$ at $300 \mathrm{~K}$. Each curve represents an isomer, and the corresponding geometry is labeled next to it. Color code for ball-stick model: Pt - blue; C - grey, O - red, H - white.

The complexity brought about by the potential dependence originates in the different potential-dependence of each isomer for each M. In this study, the electronic free energypotential relation is approximated by a capacitance model with parabolic shape where the location of the maximum corresponds to the isomer-specific potential of zero charge (PZC) 
and the curvature indicates the polarizability of the system (all curves provided in Figure S3). ${ }^{16}$ As these two properties vary among cluster isomers, the parabolic functions and isomer populations exhibit crossovers. The isomers can differ by: (i) configuration of the metal core, (ii) adsorbate configurations (orientation and bonding mode), and (iii) clustersupport interaction. For example, although the core structure of $\mathrm{Pd}_{4} / \mathrm{C}$ remains tetrahedral for all reaction intermediates (Figure S4), the cluster-surface interaction (Figure S4a) and adsorbate configurations (Figure S4b) diversify the ensemble. There are also cases where a specific isomer dominates in the whole ORR potential window, such as $\mathrm{Pd}_{4}{ }^{*} \mathrm{OH} / \mathrm{C}$ (Figure S4c), $\mathrm{Pd}_{4}{ }^{*} \mathrm{OOH} / \mathrm{C}$ (Figure S4d), $\mathrm{Ag}_{4}{ }^{*} \mathrm{O} / \mathrm{C}$ (Figure S5b), and $\mathrm{Au}_{4} / \mathrm{C}$ (Figure S6a), and cases where multiple isomers coexist in a wide potential range, such as $\mathrm{Pt}_{4}{ }^{*} \mathrm{OOH} / \mathrm{C}$ (Figure 2d), $\mathrm{Au}_{4}{ }^{*} \mathrm{OH} / \mathrm{C}$ (Figure S6c). Note that we assume the fully Boltzmann ensemble, i.e., that barriers to cluster isomerization are small and easily crossed in reaction conditions. ${ }^{17,26}$ The ensemble size is determined by the architecture of the potential-dependent free energy surface, which cannot be expressed in a unified model based on simple elemental properties, nor predicted without performing global optimization for every reaction intermediate.

The ensemble-averaged $\Delta E_{\mathrm{O}}-\Delta E_{\mathrm{OH}}$ in the potential range of 0 to $1.23 \mathrm{~V}_{\mathrm{SHE}}$ can shift away from the constant-charge DFT results by as much as $1 \mathrm{eV}$, and the shifting direction is metal-dependent (Figure 1d). The free energy change of each ORR step, $\Delta G_{i}(U)(i=1$ 4), following the associative 4-electron pathway: 
$(1) *+\mathrm{O}_{2}+\mathrm{H}^{+}+\mathrm{e}^{-}==* \mathrm{OOH}$,

(2) $* \mathrm{OOH}+\mathrm{H}^{+}+\mathrm{e}^{-}={ }^{*} \mathrm{O}+\mathrm{H}_{2} \mathrm{O}$,

$(3) * \mathrm{O}+\mathrm{H}^{+}+\mathrm{e}^{-}={ }^{*} \mathrm{OH}$,

(4) $* \mathrm{OH}+\mathrm{H}^{+}+\mathrm{e}^{-}={ }^{*}+\mathrm{H}_{2} \mathrm{O}$,

in the potential range of 0 to $1.23 \mathrm{~V}_{\mathrm{SHE}}$ for the studied $\mathrm{M}_{4} / \mathrm{C}$ systems are shown in Figure 3. It is clear that the reaction free energies do not follow the usually assumed linear potential-dependence of $1 \mathrm{eV}$ per $\mathrm{V}^{18}$ - most of them are not linear at all, with each bending in the curves corresponding to a crossover in the isomer populations in Figure 2. 

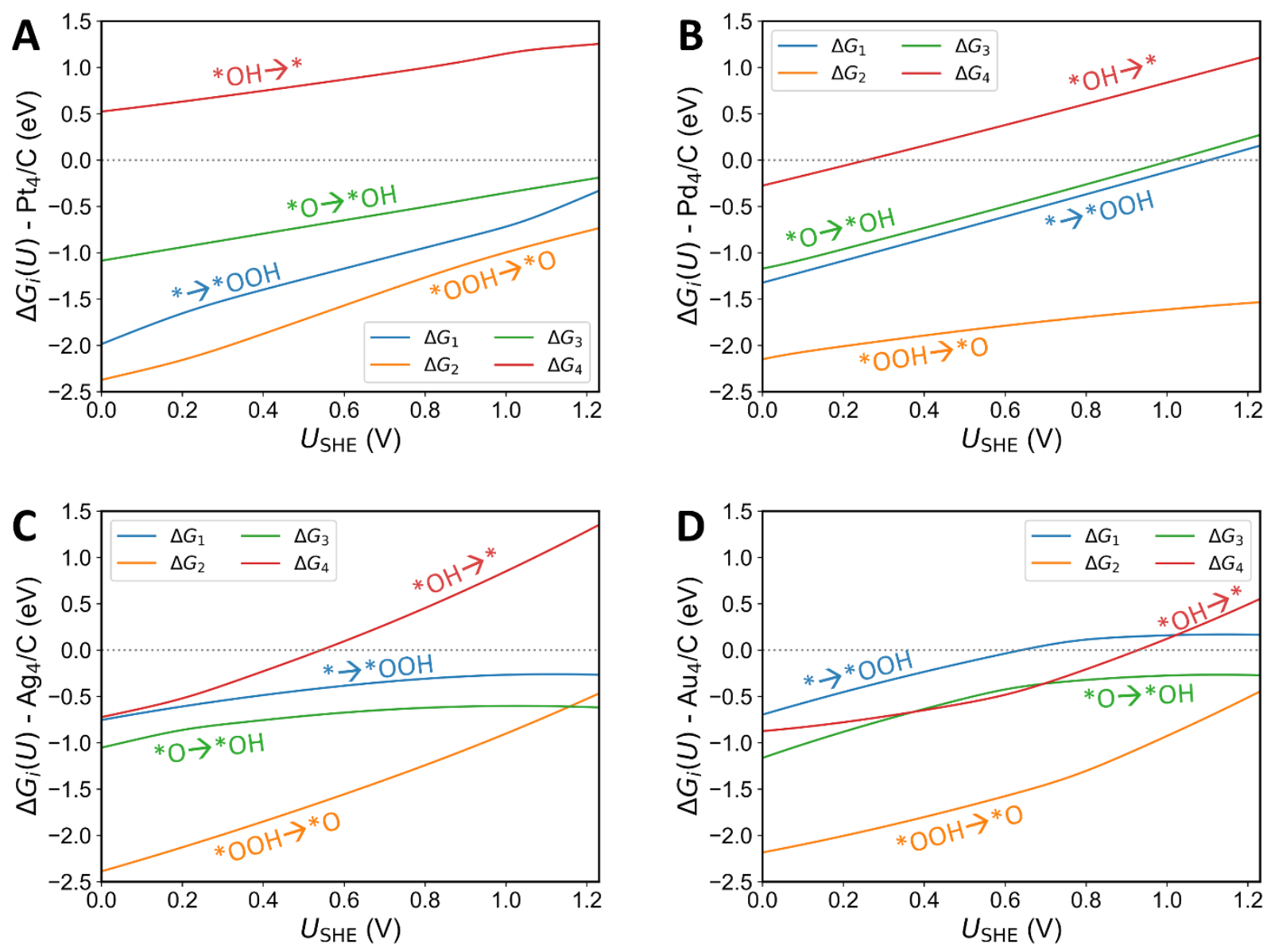

Figure 3. The potential-dependent reaction free energies of steps 1-4 of the 4-electron associative ORR pathway for (a) $\mathrm{Pt}_{4} / \mathrm{C}$, (b) $\mathrm{Pd}_{4} / \mathrm{C}$, (c) $\mathrm{Ag}_{4} / \mathrm{C}$, and (d) $\mathrm{Au}_{4} / \mathrm{C}$. A simplified chemical equation for each step is labeled along the corresponding curve.

The overall trend of stronger binding to clusters persists, and the step 4 (red curve, ${ }^{*} \mathrm{OH}$ desorption) becomes the potential-determining step (PDS) of the whole pathway for all $\mathrm{M}_{4} / \mathrm{C}$ systems except for $\mathrm{Au}_{4} / \mathrm{C}$. Au and $\mathrm{Ag}$, which have difficulty activating $\mathrm{O}_{2}$ as bulk (111), now have much more favorable $\Delta G_{1}$. As a result, the onset potential (potential at which all $\Delta G_{i}$ become negative) for $\mathrm{Ag}_{4} / \mathrm{C}$ and $\mathrm{Au}_{4} / \mathrm{C}$ shifted to much more positive 
values, thanks to the strengthening of $* \mathrm{OOH}$ binding. However, $\mathrm{Pd}_{4} / \mathrm{C}$ and $\mathrm{Pt}_{4} / \mathrm{C}$ suffer from severe ${ }^{*} \mathrm{OH}$ over-binding (step 4 ), and the expected onset potentials are much more negative compared to those of $\mathrm{Au}_{4} / \mathrm{C}$ and $\mathrm{Ag}_{4} / \mathrm{C}$.

The found effects reshape the ORR activity volcano as follows. Recall that for the bulk (111) terrace, Pt and Pd are near the apex, while Ag and Au are located far below on the right arm of the volcano (Figure 4a). In the regime of isolated subnano clusters (Figure 4b), the strengthening of binding causes a pronounced overall shift of the metal elements, bringing $\mathrm{Ag}$ and $\mathrm{Au}$ to the apex of the volcano while kicking $\mathrm{Pt}$ and $\mathrm{Pd}$ downhill along the left arm of the volcano. In addition, the fluxionality and dynamics of subnano clusters break the linear scaling relationships that define the shape of the volcano, creating a rough landscape. Finally, for graphite-supported $\mathrm{M}_{4}$ clusters (Figure 4c), the shifting still qualitatively persists. When the electrode potential is included, the potential-dependent isomer distribution opens up an additional dimension for maximizing the ORR activity, enabling the supported subnano clusters to go beyond the volcano by populating certain isomers with optimal energetics at a certain electrode potential.

Note that the ORR activity in this study is calculated using kinetics prefactor fitted to experiments on bulk (111) in ref ${ }^{8}$, whereas the isolated and supported subnano clusters would have a higher density of active sites and electrochemically active surface area (ECSA), at a similar or lower metal loading. ${ }^{19}$ Therefore, we would expect the measured ORR activity (in terms of onset potential and half-wave potential) to be significantly 
higher for subnano clusters than for single crystal surfaces or nanoparticles, despite a similar calculated ORR activity.
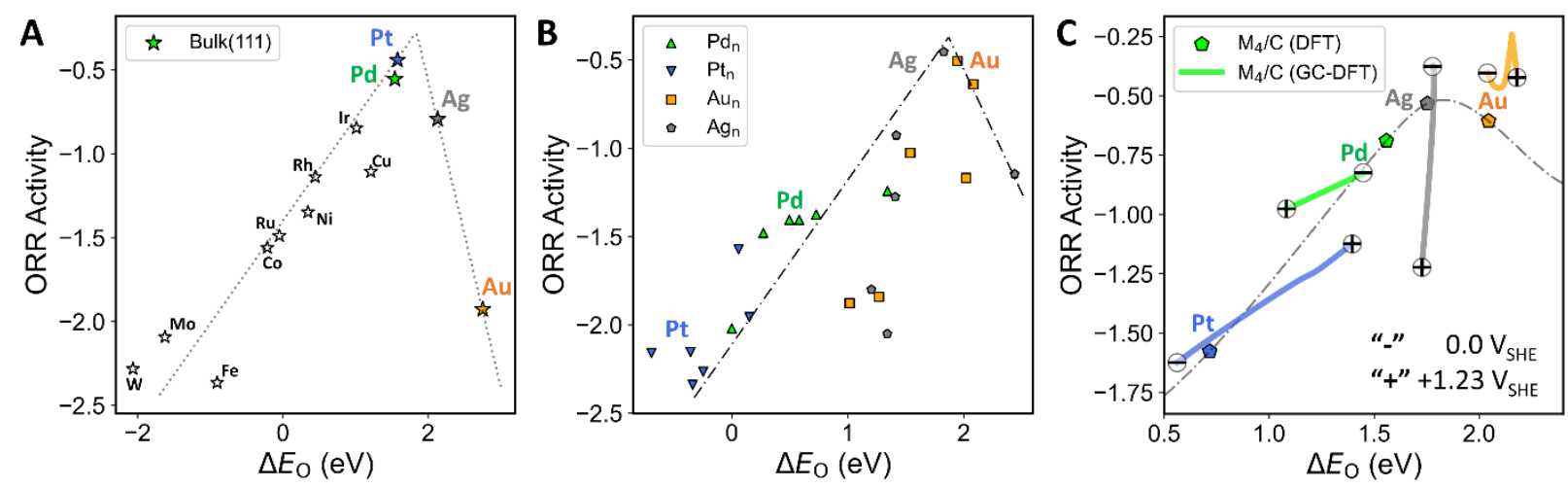

Figure 4. The ORR activity volcano for (a) bulk (111) surfaces, (b) isolated $\mathrm{M}_{1-6}$ clusters, and (c) graphite-supported $\mathrm{M}_{4}$ clusters. "+" and "_" mark the $0 \mathrm{~V}_{\text {SHE }}$ and $1.23 \mathrm{~V}_{\text {SHE values }}$ in the GCDFT curve. The $\mathrm{qU}$ term is subtracted from GCDFT energetics when calculating the activity in (c) to show the non-CHE part of the potential-dependence. Dotted and dash-dot curves are analytical volcano shape for bulk (111) in ref 8 and putative volcano shape for subnano clusters in this study, respectively.

Considering the potential-dependence of ORR activity, it is not clear which composition would correspond to the actual new apex of the volcano, because $\mathrm{Au}_{4} / \mathrm{C}$ outperforms $\mathrm{Ag}_{4} / \mathrm{C}$ and there is no datapoint beyond $\mathrm{Au}$ in the scale of $\Delta E_{\mathrm{O}}$. Possibilities include: (i) the apex being between Ag and Au, which can be accessed by alloying or heteroatom- 
doping (which we currently probe); (ii) seventh period elements (though those are rare and radioactive).

Sintering of single atoms and subnano clusters into nanostructures has been observed after electrochemical cycling, ${ }^{20,21}$ and this poses a challenge to the long-term stability of the supported subnano clusters, especially those binding to the support more weakly, such as $\mathrm{Ag}$ and $\mathrm{Au}$ (see Figure S5c-d and Figure S6c). One approach to mitigate sintering is to use a conductive substrate with appropriate anchoring sites, such as graphdiyne, black phosphorous, nanocarbon with engineered defects, and conducting metal oxides. ${ }^{22-24}$ While stronger cluster-support interaction will reshape the PES of the cluster and affect the cluster-support charge transfer, ${ }^{25-27}$ we expect the trends across fluxional clusters toward increased activity of more weakly binding metals to hold true, because fluxionality is characteristic of clusters also on more strongly binding surfaces.

In this study, to compare with the reported adsorbate binding energies on bulk (111) at $1 / 4$ monolayer coverage, we only consider one adsorbate on the $\mathrm{M}_{4} / \mathrm{C}$ systems. However, at higher current density and higher reagent concentration, there could be more adsorbates on the cluster; the binding energy and site of the second and subsequent adsorbates will depend on the identity of the previous adsorbates, ${ }^{5}$ yielding an exponentially growing number of possible configurations. Some adsorbates may remain bound to the cluster throughout the catalyzed reaction, effectively altering the stoichiometry of the catalyst to, e.g., partial oxide, hydroxide, or hydride. However, we 
expect such effect to be relatively minor for the investigated noble metals, and the fluxionality to be maintained even under stoichiometric changes, and so the observed shifting as compared to bulk surfaces should hold even at a higher coverage of mixed adsorbates.

\section{CONCLUSIONS}

In summary, we show that the apex of the ORR activity volcano is shifted from $\mathrm{Pd} / \mathrm{Pt}$ to $\mathrm{Ag} / \mathrm{Au}$ in the regime of subnano clusters due to their fluxionality. A structural ensemble representing each cluster constantly changes in the reaction, as the cluster isomerizes to maximize the adsorbate binding, with the configurations differing metal-by-metal, and adsorbate-by-adsorbate. The linear scaling relationship is hence broken. The adsorbate binding energies overall strengthen on fluxional clusters, making $\mathrm{O}_{2}$ activation no longer a barrier for $\mathrm{Ag} / \mathrm{Au}$ while giving $\mathrm{Pt} / \mathrm{Pd}$ a severe over-binding problem. Grand canonical DFT calculations are performed to construct the potential-dependent ensemble representation of supported subnano clusters. Change in electrode potential within the ORR potential window can alter the isomer distribution, making the relationship between reaction free energies and electrode potential highly non-linear, and that enables surpassing the bulk volcano apex by populating isomers with optimal energetics. Overall, we expect cluster fluxionality to quite generally shift the volcano for cluster catalysis to the left, making under-binding elements more catalytic. 


\section{METHODS}

The isolated subnano clusters are modeled in a large unit cell of $20 \AA \times 20 \AA \times 20 \AA$. The graphite substrate is modeled by a $(6 \times 6 \times 3)$ graphite slab, with the bottom two layers constrained to represent bulk behaviour and a vacuum slab of $25 \AA$ thickness. The isomer and adsorbate configurations in both isolated and supported forms are sampled using the bond length distribution algorithm (BLDA) as implement in our open source Python package, PGOPT. The local optimization and energy evaluation of the generated structures are performed with the PBE functional ${ }^{28}$ and PAW pseudopotentials ${ }^{29}$ using the VASP program (version 5.4.1). ${ }^{30-33}$ DDsC correction is used to account for the dispersion interactions. ${ }^{34}$ Implicit solvation model (solvent: water) is used for all calculations using the VASPsol code. ${ }^{35}$ Spin polarization is turned on throughout. Due to the relatively large system and sampling size, only the $\Gamma k$-point is sampled in the reciprocal space of the Brillouin zone, and the cutoff energy for the kinetic energy of the plane-waves was $400 \mathrm{eV}$. The convergence criteria are set to $10^{-6} \mathrm{eV}$ for SCF iteration and $0.05 \mathrm{eV} / \AA$ for the forces. The grand canonical DFT calculations employ the surface charging technique reported in ref ${ }^{16}$, using a symmetrized slab with a $25 \AA$ vacuum gap. The adsorbate binding energies, reaction free energies, and ORR activity are calculated using the expressions in ref ${ }^{8}$, but the energies are from ensemble average instead of the linear scaling relationships. 


\section{ASSOCIATED CONTENT}

Supporting Information. Isomer distribution of $\mathrm{M}_{4}$ and $\mathrm{M}_{4} / \mathrm{C}$ systems; Pair-correlation heatmap of adsorbate binding energies with atomic and bulk properties; Free energypotential curves from GCDFT calculations; Potential-dependent free energy and isomer distributions at $300 \mathrm{~K}, 1000 \mathrm{~K}$.

\section{AUTHOR INFORMATION}

Corresponding Author

*E-mail: ana@chem.ucla.edu

Present Addresses

${ }^{\dagger}$ Julen Munarriz - Present address: Departamento de Química Física y Analítica, Universidad de Oviedo, Oviedo, 33006, Spain

Notes

The authors declare no competing financial interests.

\section{ACKNOWLEDGMENT}

We acknowledge the support from the U.S. Department of Energy grant DE-SC0020125 to A. N. A. The project used the computational resources at NERSC and UCLA shared cluster, Hoffman2. 


\section{REFERENCES}

(1) Bligaard, T.; Nørskov, J. K.; Dahl, S.; Matthiesen, J.; Christensen, C. H.; Sehested, J. The Brønsted-Evans-Polanyi Relation and the Volcano Curve in Heterogeneous Catalysis. J. Catal. 2004, 224, 206-217.

(2) Ooka, H.; Huang, J.; Exner, K. S. The Sabatier Principle in Electrocatalysis: Basics, Limitations, and Extensions. Front. Energy Res. 2021, 9, 155.

(3) Huang, Z.-F.; Song, J.; Dou, S.; Li, X.; Wang, J.; Wang, X. Strategies to Break the Scaling Relation toward Enhanced Oxygen Electrocatalysis. Matter 2019, 1, 14941518.

(4) Zhai, H.; Alexandrova, A. N. Fluxionality of Catalytic Clusters: When It Matters and How to Address It. ACS Catal. 2017, 7, 1905-1911.

(5) Zhang, Z.; Zandkarimi, B.; Alexandrova, A. N. Ensembles of Metastable States Govern Heterogeneous Catalysis on Dynamic Interfaces. Acc. Chem. Res. 2020, 53, $447-458$.

(6) Zandkarimi, B.; Alexandrova, A. N. Dynamics of Subnanometer Pt Clusters Can Break the Scaling Relationships in Catalysis. J. Phys. Chem. Lett. 2019, 10, 460467.

(7) Zandkarimi, B.; Alexandrova, A. N. Can Fluxionality of Subnanometer Cluster 
Catalysts Solely Cause Non-Arrhenius Behavior in Catalysis? J. Phys. Chem. C 2020, 124, 19556-19562.

(8) Nørskov, J. K.; Rossmeisl, J.; Logadottir, A.; Lindqvist, L.; Kitchin, J. R.; Bligaard, T.; Jónsson, H. Origin of the Overpotential for Oxygen Reduction at a Fuel-Cell Cathode. J. Phys. Chem. B 2004, 108, 17886-17892.

(9) Zhai, H.; Alexandrova, A. N. Ensemble-Average Representation of Pt Clusters in Conditions of Catalysis Accessed through GPU Accelerated Deep Neural Network Fitting Global Optimization. J. Chem. Theory Comput. 2016, 12, 6213-6226.

(10) Markovic, N. R.; Ross, P. N. New Electrocatalysts for Fuel Cells from Model Surfaces to Commercial Catalysts. Cattech 2000, 4, 110-126.

(11) Valden, M.; Lai, X.; Goodman, D. W. Onset of Catalytic Activity of Gold Clusters on Titania with the Appearance of Nonmetallic Properties. Science (80-. ). 1998, 281, 1647-1650.

(12) Heiz, U.; Sanchez, A.; Abbet, S.; Schneider, W.-D. Catalytic Oxidation of Carbon Monoxide on Monodispersed Platinum Clusters: Each Atom Counts. J. Am. Chem. Soc. 1999, 121, 3214-3217.

(13) Tsunoyama, H.; Ohnuma, A.; Takahashi, K.; Velloth, A.; Ehara, M.; Ichikuni, N.; Tabuchi, M.; Nakajima, A. Enhanced Oxygen Reduction Activity of Platinum 
Subnanocluster Catalysts through Charge Redistribution. Chem. Commun. 2019, $55,12603-12606$.

(14) Steinmann, S. N.; Sautet, P. Assessing a First-Principles Model of an Electrochemical Interface by Comparison with Experiment. J. Phys. Chem. C2016, $120,5619-5623$.

(15) Steinmann, S. N.; Michel, C.; Schwiedernoch, R.; Filhol, J.; Sautet, P. Modeling the $\mathrm{HCOOH} / \mathrm{CO} 2$ Electrocatalytic Reaction: When Details Are Key. ChemPhysChem $2015,16,2307-2311$.

(16) Steinmann, S. N.; Michel, C.; Schwiedernoch, R.; Sautet, P. Impacts of Electrode Potentials and Solvents on the Electroreduction of CO 2: A Comparison of Theoretical Approaches. Phys. Chem. Chem. Phys. 2015, 17, 13949-13963.

(17) Imaoka, T.; Toyonaga, T.; Morita, M.; Haruta, N.; Yamamoto, K. Isomerizations of a Pt 4 Cluster Revealed by Spatiotemporal Microscopic Analysis. Chem. Commun. 2019, 55, 4753-4756.

(18) Alfonso, D. R.; Tafen, D. N.; Kauffmann, D. R. First-Principles Modeling in Heterogeneous Electrocatalysis. Catalysts 2018, 8, 424.

(19) Tang, Z.; Wu, W.; Wang, K. Oxygen Reduction Reaction Catalyzed by Noble Metal Clusters. Catalysts 2018, 8, 65. 
(20) Weng, Z.; Wu, Y.; Wang, M.; Jiang, J.; Yang, K.; Huo, S.; Wang, X. F.; Ma, Q.;

Brudvig, G. W.; Batista, V. S.; Liang, Y.; Feng, Z.; Wang, H. Active Sites of Copper-Complex Catalytic Materials for Electrochemical Carbon Dioxide Reduction. Nat. Commun. 2018, 9, 1-9.

(21) Corma, A.; Concepción, P.; Boronat, M.; Sabater, M. J.; Navas, J.; Yacaman, M. J.; Larios, E.; Posadas, A.; López-Quintela, M. A.; Buceta, D. Exceptional Oxidation Activity with Size-Controlled Supported Gold Clusters of Low Atomicity. Nat. Chem. 2013, 5, 775-781.

(22) Liu, J.-C.; Xiao, H.; Li, J. Constructing High-Loading Single-Atom/Cluster Catalysts via an Electrochemical Potential Window Strategy. J. Am. Chem. Soc. $2020,142,3375-3383$.

(23) Chia, X.; Pumera, M. Characteristics and Performance of Two-Dimensional Materials for Electrocatalysis. Nat. Catal. 2018, 1, 909-921.

(24) von Weber, A.; Baxter, E. T.; Proch, S.; Kane, M. D.; Rosenfelder, M.; White, H. S.; Anderson, S. L. Size-Dependent Electronic Structure Controls Activity for Ethanol Electro-Oxidation at Ptn/Indium Tin Oxide ( $\mathrm{n}=1$ to 14$)$. Phys. Chem. Chem. Phys. 2015, 17, 17601-17610.

(25) Jimenez-Izal, E.; Zhai, H.; Liu, J.-Y.; Alexandrova, A. N. Nanoalloying MgODeposited Pt Clusters with Si To Control the Selectivity of Alkane 
Dehydrogenation. ACS Catal. 2018, 8, 8346-8356.

(26) Zhai, H.; Alexandrova, A. N. Local Fluxionality of Surface-Deposited Cluster Catalysts: The Case of Pt7 on Al2O3. J. Phys. Chem. Lett. 2018, 9, 1696-1702.

(27) Baxter, E. T.; Ha, M.-A.; Cass, A. C.; Zhai, H.; Alexandrova, A. N.; Anderson, S. L. Diborane Interactions with Pt7/Alumina: Preparation of Size-Controlled Borated Pt Model Catalysts. J. Phys. Chem. C2018, 122, 1631-1644.

(28) Adamo, C.; Barone, V. Toward Reliable Density Functional Methods without Adjustable Parameters: The PBE0 Model. J. Chem. Phys. 1999, 110, 6158-6170.

(29) Kresse, G.; Joubert, D. From Ultrasoft Pseudopotentials to the Projector Augmented-Wave Method. Phys. Rev. b 1999, 59, 1758.

(30) Kresse, G.; Furthmüller, J. Efficiency of Ab-Initio Total Energy Calculations for Metals and Semiconductors Using a Plane-Wave Basis Set. Comput. Mater. Sci. $1996,6,15-50$.

(31) Kresse, G.; Furthmüller, J. Efficient Iterative Schemes for Ab Initio Total-Energy Calculations Using a Plane-Wave Basis Set. Phys. Rev. B 1996, 54, 11169-11186.

(32) Kresse, G.; Hafner, J. Ab Initio Molecular Dynamics for Liquid Metals. Phys. Rev. $B 1993,47,558$. 
(33) Kresse, G.; Hafner, J. Ab Initio Molecular-Dynamics Simulation of the LiquidMetal-Amorphous-Semiconductor Transition in Germanium. Phys. Rev. B 1994, $49,14251$.

(34) Steinmann, S. N.; Corminboeuf, C. A Generalized-Gradient Approximation Exchange Hole Model for Dispersion Coefficients. J. Chem. Phys. 2011, 134, 44117.

(35) Mathew, K.; Sundararaman, R.; Letchworth-Weaver, K.; Arias, T. A.; Hennig, R. G. Implicit Solvation Model for Density-Functional Study of Nanocrystal Surfaces and Reaction Pathways. J. Chem. Phys. 2014, 140, 84106. 